\title{
Systematization and clinical study of ' dextroversion, mirror-image dextrocardia, and laevoversion
}

\author{
Guillermo Anselmi, Simon Muñoz, Pablo Blanco, Ivan Machado, \\ and María V. de la Cruz \\ - From the Department of Paediatric Cardiology, Hospital Universitario, Caracas, Venezuela; \\ and the Laboratory of Embryology, Instituto Nacional de Cardiologia, México
}

- Current embryological and anatomical knowledge makes it possible to systematize, classify, and diagnose the malpositions of the heart by following a logical sequence. (I) The visceral situs is easily established by means of data provided by physical, radiological, and electrocardiographic studies. There is a constant relation between the venous atrium, greater lobe of the liver with suprahepatic segment of the inferior vena cava, and the visceral situs. (2) The direction of the

- apex: this indicates the heart position in a specific situs, determined by x-rays. Dextroversion is the malposition in situs solitus, as laevoversion is in situs inversus; dextrocardia is the normal position for the latter. (3) Truncoconal morphologies: there are 3 possibilities (a) the vessels

- derived from the truncus conus are not transposed, (b) the vessels are transposed, or (c) there is a common trunk. Truncoconal morphology is determined in each case by means of a biplane

- angiocardiogram. (4) The position of the ventricles is deduced from the truncoconal morphology, by applying the rule which establishes whether the anterior vessel or single trunk emerges from the ventral ventricle; correlating the position of this ventricle with the situs, we find out

- whether or not there is ventricular inversion. (5) Associated malformations are diagnosed by clinical as well as haemodynamic studies, completing finally the diagnosis in every case.

As stated in a previous paper (De la Cruz et al., 1971), present knowledge enables us to establish an interrelation between the visceral situs, truncoconal morphology, and the position of the chambers of the heart, which leads , us to a sufficiently precise understanding of

- the malpositions, a logical classification, and

- a rational diagnosis.

Out of 3000 cases of congenital heart dis( ease studied at the Hospital Universitario of Caracas, we have selected 4I malpositions of the heart $(1 \cdot 3 \%)$; of these, 19 were dextro-

- cardias, 7 were laevoversions, and 12 were dextroversions; these cases were studied by

- haemodynamic and angiocardiographic examinations and ro of them by necropsy.

On the basis of current knowledge of the development of the heart as well as on examination of necropsy specimens, the clinical diagnosis of malpositions of the heart is considerably simplified if a logical and $\rightarrow$

Received 28 January 1972. ordered sequence is adopted (Anselmi et al., 1970): (I) the visceral situs, (2) the direction of the apex, (3) the truncoconal morphologies, (4) the position of the ventricles, and (5) associated defects.

\section{Diagnosis of the visceral situs}

The visceral situs is established in the embryo at a very early stage. Our diagnosis is based on the constant relation presented for each situs by: the venous atrium, ${ }^{1}$ the greater lobe of the liver, and the suprahepatic segment of the inferior vena cava; there is, therefore, a constant hepato-cavo-atrial concordance for each situs. Other visceral and anatomical formations may present variations in their position within a given situs, and we do not,

\footnotetext{
1 To avoid confused expressions we will call in this paper venous atrium the atrial cavity characterized anatomically by the fossa ovalis, the crista terminalis, and the pectineus muscles, which normally receive the venae cavae and the coronary sinus; it corresponds to the so-called anatomically right atrium.
} 
for this reason, take them into account in our diagnosis, as they are variable factors which might lead to confusion. In practice, it is difficult to take into account the suprahepatic segment of the inferior vena cava in diagnosing the visceral situs, owing to the difficulty of visualizing this vessel except by angiographic examination, in which case it is sufficient to locate the greater lobe of the liver and the venous atrium.

There are three types of situs:

a) Situs solitus, in which the greater lobe of the liver, the venous atrium, and the suprahepatic segment of the inferior vena cava are on the right.

b) Situs inversus, in which the greater lobe of the liver, the venous atrium, and the suprahepatic segment of the inferior cava are on the left.

c) The indefinite situs, where the liver is centrally situated, has two lobes of equal size; there is atrial isomerism and heterotaxia of the abdominal viscerae. ${ }^{2}$

The situs is easily diagnosed by palpation and percussion of the liver as well as by radiological examination of the hepatic shadow. It must be kept in mind that newborn and premature infants have large livers, in a fairly central position, but in most cases it is possible to locate the greater lobe, especially by $x$-ray examination.

The position of the venous atrium is generally determined by the electrocardiogram. This shows the spatial axis of the $P$ wave (SÅP) directed towards the front, downwards, and to the left in situs solitus (Portillo et al., 1959), and towards the front, downwards, and to the right in situs inversus. The factor of error or doubt in some cases could be due to atrial ectopic rhythms, atrial fibrillation, or flutter. Nodal rhythm in situs solitus shows the frontal ÂP directed upwards and to the left $\left(-60^{\circ}\right)$ and upwards and to the right $\left(+120^{\circ}\right)$ in situs inversus.

The passage of a catheter through the inferior vena cava would lead us to the venous atrium and would indicate its position; when the catheter is inserted through the saphenous vein, we are able to determine the position of the suprahepatic segment of the inferior vena cava. In some fairly frequent types of malposition, there are anomalies of systemic venous return, such as partial absence of the inferior vena cava, which make it difficult to determine the suprahepatic segment of this vessel; in such cases it is the venous angiogram that will indicate the position of 2 The indefinite situs and displacements of the heart due to extracardiac causes will not be discussed here.

the venous atrium. In practice, the location of the greater lobe of the liver and of the venous atrium are easy to determine by physical examination, $x$-rays, and the electrocardiogram.

\section{Diagnosis of direction of apex}

In a given situs, the position of the apex indicates the type of malposition. In situs solitus there are two possible positions (Fig. I). (a) Apex on the left: normally situated heart. (b) Apex on the right: dextroversion.

FIG. I The two possibilities in situs solitus: apex to the left, which corresponds to the normally situated heart and apex to the right, which corresponds to dextroversion. Electrocardiogram: note the $P$ wave of low voltage but positive in lead I and the morphologies with deep $Q$ waves in leads $I$ and II. The voltage of the ventricular complexes decreases from VI through V6. The $q R$ morphologies corresponding to variations of potential of the dorsal ventricle are recorded from $V_{3}$ to V6; $r S$ morphologies corresponding to variations of potential of the ventral ventricle are recorded in the right praecordial leads (V $2 R$ to $V 5 R$ ), thus suggesting a normal ventricular position, concordant with the situs. X-rays show the shadow of the greater lobe of the liver on the right, the heart in the right hemithorax, and the apex directed towards the right. Both studies (electrocardiogram and $\mathrm{x}$-rays) correspond to the case of an infant with dextroversion without associated defects.

$V A$ : venous atrium; $A A$ : arterial atrium.

\section{SITUS SOLITUS}

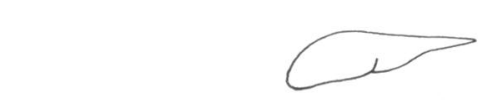

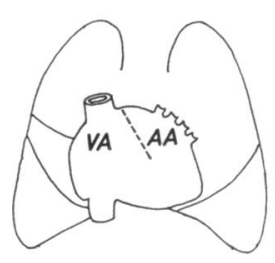

NORMALLY SITUATED HEART

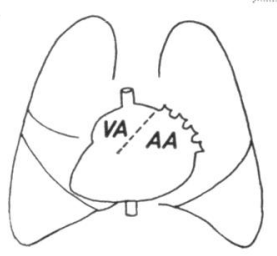

DEXTROVERSION

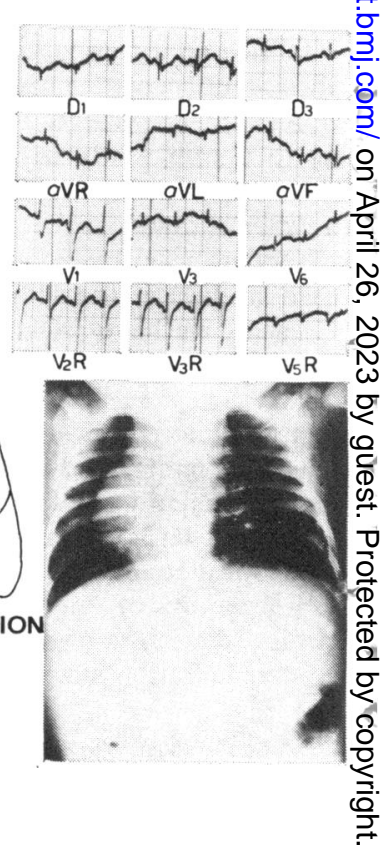


In situs inversus there are two positions of the heart. (a) Apex to the right: mirror-image - or simply dextrocardia; it is the normal position of the heart for situs inversus. (b) Apex to the left: laevoversion, an abnormal position for situs inversus.

The diagnosis of the direction of the apex is easy to establish in most cases by clinical examination. Inspection and palpation of the - apex in the right hemithorax; the cardiac sounds louder on the right side of the ster- num than on the left, and percussion of an area of dullness on that side enables us to diagnose dextroversion in situs solitus. $X$-rays indicate the direction of the apex and show the cardiac shadow on the right. The electrocardiogram shows certain characteristics of this malposition: $\mathbf{P}$ waves in lead $\mathrm{I}$ are of low voltage, usually diphasic of the \pm type; followed by ventricular complexes of the $Q R$ type in lead I or II; the transitional zone is displaced to the right and transitional RS complexes can be seen in VI; morphologies of the $\mathrm{qR}$ or QR type are found in V5 and V6,

FIG. 2 The two possibilities in situs inversus; apex to the right which corresponds to

- dextrocardia. Electrocardiogram: the $P$ wave is negative in lead I and positive in aVR. The $\hat{A P}$ is directed downward, forward, and to the right, which indicates the position of the sinuatrial

- node to the left (and hence of the venous atrium). The $q R$ morphologies in V6R correspond to variations of potential of the dorsal ventricle; $r S$ morphologies from VI to V6 correspond to

- variations of potential of the ventral ventricle, thus suggesting a normal ventricular position, concordant with the situs. X-rays: the shadow of the liver is seen to the left, the heart is in the right hemithorax, with the apex directed towards the right. Both studies (electrocardiogram and $\mathrm{x}$-rays) correspond to the case of an adult with dextrocardia without associated defects.

Apex to the left which corresponds to laevoversion. Electrocardiogram: the negative $P$ wave

in lead I and still more negative in aVL than in aVR, with the AP directed downward, forward, and to the right indicates the position of the sinuatrial node to the left (and hence of the venous

- atrium). The analysis of the ventricular morphologies does not allow determination of the ventricular position.

X-rays: the shadow of the liver is seen to the left, the heart is in the left hemithorax and the apex is directed towards the left. These studies correspond to the case of a patient with

- laevoversion, single ventricle, and pulmonary stenosis.
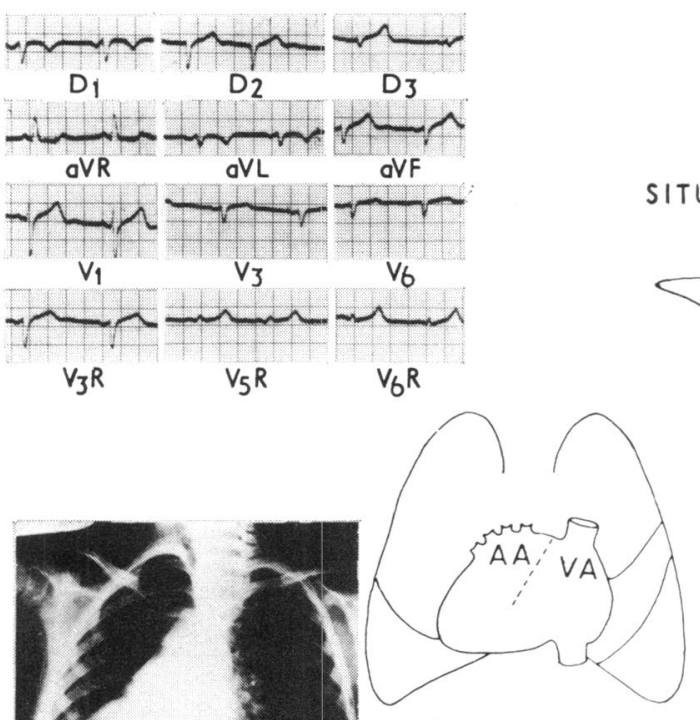

DEXTROCARDIA

\section{SITUS INVERSUS}

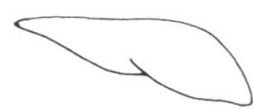

LAEVOVERSION

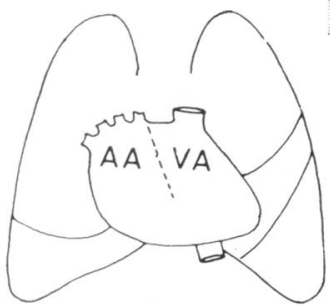

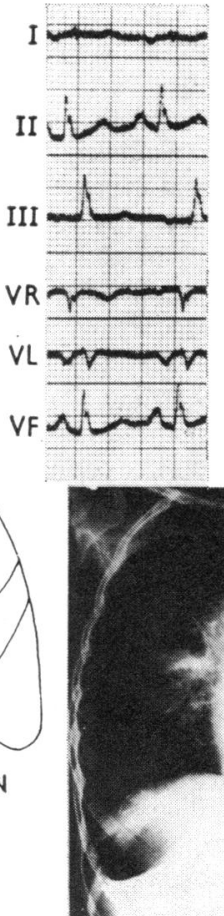

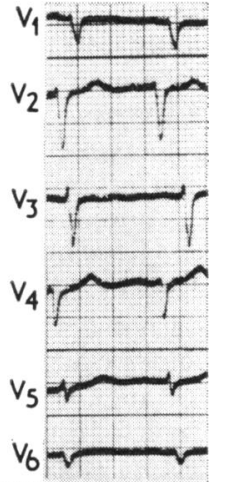


which indicate variations of potential of the 'left atrium', the electrodes corresponding to these leads being oriented towards this cavity (Sodi Pallares et al., 1959); these potentials are also found in aVL and, consequently, in lead I. The voltage of the ventricular complexes diminishes progressively from VI to V6 and increases in the direction of the right praecordial leads $V_{4} R$ to $V 6 R$, owing to the proximity of the heart to the latter leads (Fig. I).

In situs inversus, similarly, examination and palpation of the apex and percussion of an area of dullness on the right, lead us to suspect dextrocardia. $X$-rays show the direction of the apex and the cardiac shadow on the right. The electrocardiogram helps in diagnosing dextrocardia (Fig. 2) as, besides the signs mentioned in describing the situs inversus in general, there are characteristics which enable us to suspect that the heart is in the right hemithorax: the low voltage in the left praecordial leads and its increase in those on the right (V3R to V6R), owing to the proximity of the heart to these leads; mirror-images of the normal ventricular complexes and of the $T$ wave in leads I and aVL, the complexes in these leads being predominantly negative. It is important to know the unipolar morphologies corresponding to variations of potential peculiar to the ventricles, both in normal and pathological conditions, in order to be able to arrive at a logical electrocardiographic interpretation and apply it to the diagnosis of malpositions (Sodi Pallares et al., 1959).

In situs inversus, if the examination and palpation of the apical impulse and percussion of an area of dullness on the left, and the auscultation of cardiac sounds of greater intensity to the left of the sternum, indicate that the heart is situated to the left of the sternum, the diagnosis of laevoversion becomes evident. $X$-rays show the direction of the apex and the cardiac shadow situated on the left (Fig. 2). In these cases, as well as the factors mentioned in connexion with the situs inversus, the cardiogram often shows a progressive increase in the voltage of the ventricular complexes towards the left praecordial leads and its lowering in those of the right hemithorax.

\section{Diagnosis of truncoconal morphologies}

When the visceral situs and the direction of the apex have been diagnosed, we are able to determine the type of malposition of the heart we are dealing with, and can proceed to diagnose the truncoconal morphologies, the position of the ventricles, and associated malformations.

A biplane angiocardiogram is essential for investigating the last three variations and it enables us to complete the diagnosis. However, the $x$-rays and the electrocardiogram often lead us to suspect associated defects.

The position of the ventricles is deduced from the truncoconal morphology. It is, therefore, essential to determine in the first place the position and characteristics of the great arteries. There are three basic types of truncoconal morphologies in situs inversus, with or without ventricular inversion; they are: (a) without transposition of the great arteries (TGA); (b) with transposition of the great arteries; and (c) with a common trunk (De la Cruz et al., 1967). Associated malformations as well as truncoconal morphologies and the position of the ventricles occur in malpositions with different statistical frequency - hence it is important to examine them separately.

\section{Dextroversion}

This is a fairly frequent malposition; 12 of our $4 \mathrm{I}$ cases of malposition were dextroversions $(29 \%)$. It has been described under different names: dextrorotation (Ayres and Steinberg, 1963), isolated dextrocardia (Shaher and Johnson, 1963), dextrocardia with atria in situs solitus (Calderón et al., 1965); in fact, some of the published descriptions call dextroversion other malpositions, which accounts for the confusion shown in many statistics as to the actual incidence of this malformation. We call dextroversion a malposition characterized by situs solitus (greater

FIG. 3 Scheme of the 6 possible combinations in dextroversion with and without ventricular inversion, according to the three truncoconal morphologies. VV, ventral ventricle; DoV, dorsal ventricle.

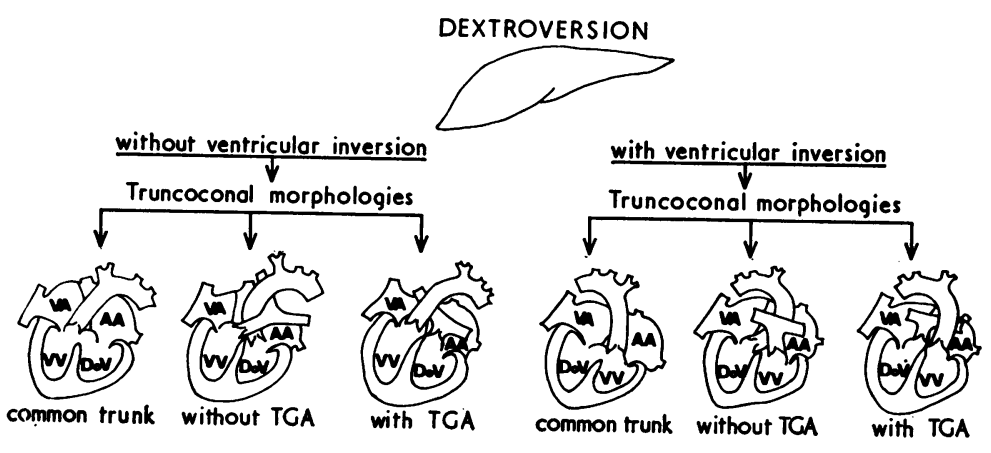


lobe of the liver, venous atrium, and the suprahepatic segment of the inferior vena - cava located on the right) with the heart in the right hemithorax and its apex directed towards the right. One should not include here other malformations such as hererotaxias (indefinite situs with dextroisomerism) with asplenia and dextropositions (displacement of the heart to the right due to extra- cardiac causes), which do not correspond to the embryological mechanism of dextroversion.

Dextroversion is complicated in 90 per cent of the cases by cardiac malformations (Grant, 1958). Only Io per cent show no evidence of cardiac abnormality; of our 12 cases, 2 - showed no associated malformations. This is probably related to the fact that dextroversion

- is an abnormal position of the heart in a situs solitus malformation that appears very early

> in development, and for this reason it is often complicated by complex malformations; 83 per cent of cases show severe cyanotic abnormalities and only 7 per cent mild and noncyanotic malformations.

Truncoconal morphologies in dextroversion are of three basic types: type (a) without

- TGA; (b) with TGA; and (c) with a common trunk in different statistical proportions; type (b) with TGA is the most common with 58 per cent of our cases and 67 per cent of those already published (Ayres and Steinberg, 1963; Calderón et al., 1965; Berri, 1958; Grant, I958; type (c) with a common trunk is the least frequent.

The type of truncoconal morphology is suggested by (I) statistical data, which make us think of TGA in 69 per cent of dextroversions; (2) widening of the vascular pedicle shown by $x$-rays in oblique incidences and - the absence of the thymus in the newborn and infants (this is often encountered, in our experience) also suggest the possibility of TGA; (3) slight or absent cyanosis, a single second sound at the base, and a soft continuous murmur, as well as the radiological picture suggestive of a common trunk, and also

- indicative; (4) the absence of cyanosis, the evidence of a mild malformation (pulmonary or aortic valvular stenosis or simple septal defects), or the absence of associated malformations, suggest type (a) without TGA.

The diagnosis of truncoconal morphology is made by means of a biplane angiocardiogram: if the vessels cross in space - the pulmonary artery (PA) being anterior and the aorta posterior - we are dealing with type (a) without TGA; if the vessels are parallel, the aorta being anterior and the pulmonary artery posterior, we have type (b) with TGA; and

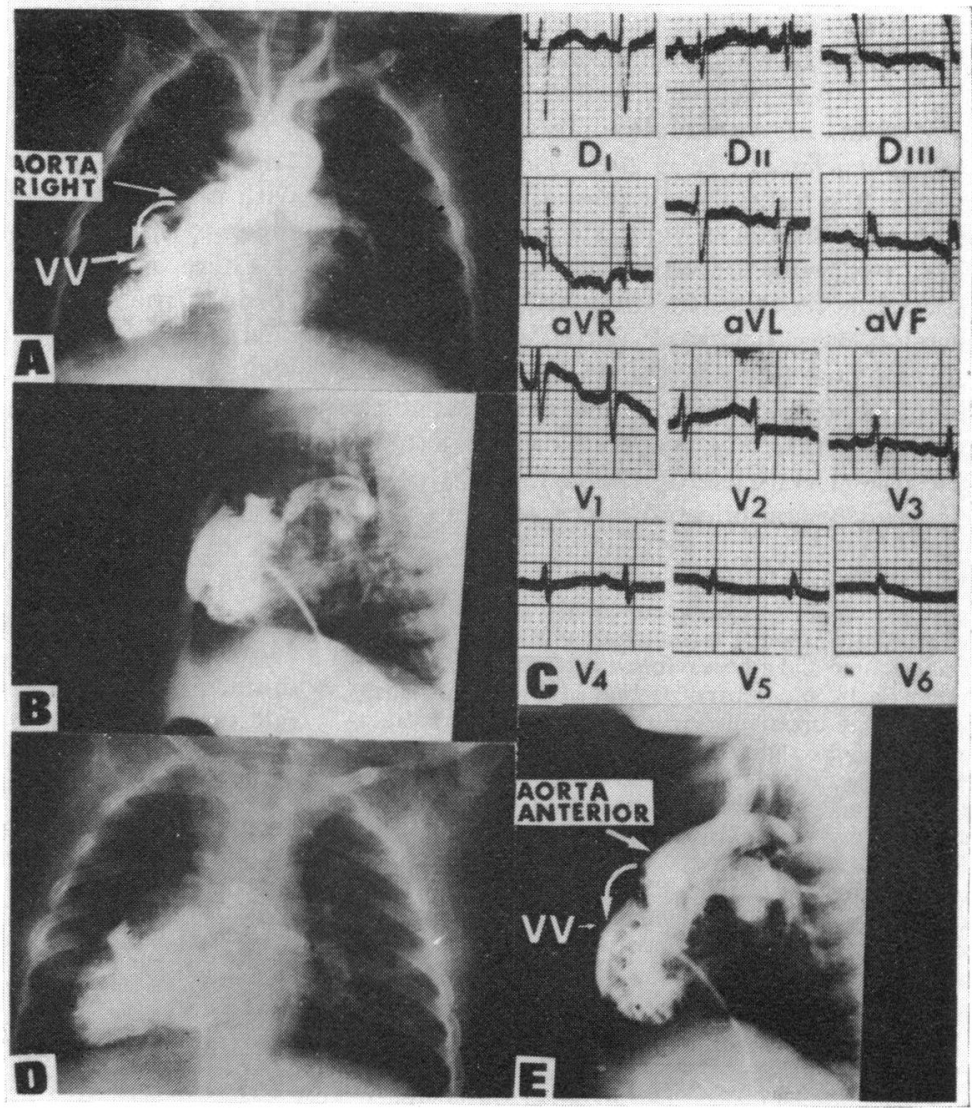

FIG. 4 Angiocardiographic and electrocardiographic studies in a case of situs solitus.

The electrocardiogram $(C)$ shows positive $P$ waves in lead $I$ and negative in aVR, suggesting that the atria are in the situs solitus position; the decrease in voltage from $V_{I}$ to $V 6$ suggests that the heart is in the right hemithorax: dextroversion. The $q R$ complexes in $V 5$ and $V 6$ correspond to variations of potential of the dorsal ventricle, situated to the left.

The obvious deviation of the AQRS to the right suggests the possibility of enlargement of the ventricle which is situated to the right.

The angiocardiogram in the lateral projection shows that the aorta is anterior, and in the frontal projection $(A)$ the same vessel is situated to the right; therefore the ventral ventricle is placed to the right, which is concordant with the situs. Further, there is evidence $(B, D, E)$ of pulmonary stenosis (unequal partition) and both vessels emerge almost entirely from the ventral ventricle (dextroposition). This is a case of situs solitus with dextroversion without ventricular inversion, TGA, pulmonary stenosis, lateroposition, and ventricular septal defect. 
if a single vessel emerges from the heart, we have type (c) with a common trunk.

Position of ventricles In dextroversion the ventricles can be in their normal positions, that is the ventral ventricle ${ }^{1}$ situated on the right and the dorsal ventricle ${ }^{2}$ situated on the left, this being the ventricular position corresponding to the situs in which the dextroversion has occurred (situs solitus). The second possibility is ventricular inversion (De la Cruz et al., 1967). This means that the ventral ventricle is situated on the left and the dorsal ventricle on the right. In 5 of our Io cases of complicated dextroversion ( $50 \%)$,

\begin{abstract}
${ }^{1}$ In this paper ventral ventricle is the ventricular chamber characterized anatomically by the presence of a supraventricular crista whose septal wall is thickly trabeculated and whose outflow chamber, derived from the cephalic and ventral portion of the bulbus cordis, occupies an invariably ventral position. It is traditionally called the right ventricle.

2 Dorsal ventricle is the ventricular chamber characterized anatomically by a smooth septal wall in its superior two-thirds and presenting in the lower third fine trabeculations, directed along the longitudinal axis of the ventricle, whose outflow chamber, derived from the cephalic and dorsal portion of the bulbus cordis, occupies an invariably dorsal position. It is traditionally called the left ventricle.
\end{abstract}

FIG. 5 In this case, the electrocardiogram shows $P$ waves of the \pm type in lead $I$ and negative in aVR, suggesting that the atria are in the situs solitus position, and the peaked and tall $P$ waves indicate right atrial overloading. The $q R$ morphologies in $V 4 R$ and $V 6 R$ suggest that the dorsal ventricle is situated to the right and the $r S$ morphologies in $V_{3}$ indicate that the ventral ventricle is situated to the left, which is abnormal for this situs, thus suggesting ventricular inversion.

The $\mathrm{x}$-rays ( $A$ and $B$ ) show the shadow of the liver to the right, the heart in the right hemithorax with the apex directed toward the right: dextroversion. The pulmonary flow is decreased, which is suggestive of pulmonary stenosis. In B the vascular pedicle is seen widened and the aorta emerges apparently anteriorly, which suggests TGA. The angiocardiogram in $D$ shows that the aorta is anterior, and in the frontal projection $(C)$ the aorta emerges to the left, thus indicating that the ventral ventricle is situated to the left ventricular position which is abnormal for this situs. There is also evidence of pulmonary stenosis (unequal partition) in D. This is a case of situs solitus with dextroversion, ventricular inversion, TGA, pulmonary stenosis, and ventricular septal defect. there was ventricular inversion, which proves the high incidence of this abnormal ventricular position in dextroversion.

The three truncoconal morphologies described above can be seen in dextroversion with or without ventricular inversion, which raises to 6 the diagnostic possibilities (Fig. 3).

There are certain facts that enable us to suspect the position of the ventricles in dextroversion: (I) the high incidence of ventricular inversion in dextroversion is a valuable statistical factor; (2) in some cases, in the absence of single ventricle (Anselmi et al., 1968), the electrocardiogram shows clearly discernible morphologies corresponding to ventricular variations of potential, which allow us to locate the position of the ventricles by studying the thoracic circle. Morphologies of qR, qRS in the left praecordium, and morphologies of the rS, Rs, rSr, rsR type in the right praecordial leads, indicate a normal position of the ventricles (Fig. 4). Conversely, morphologies corresponding to variations of potential of the 'left ventricle'

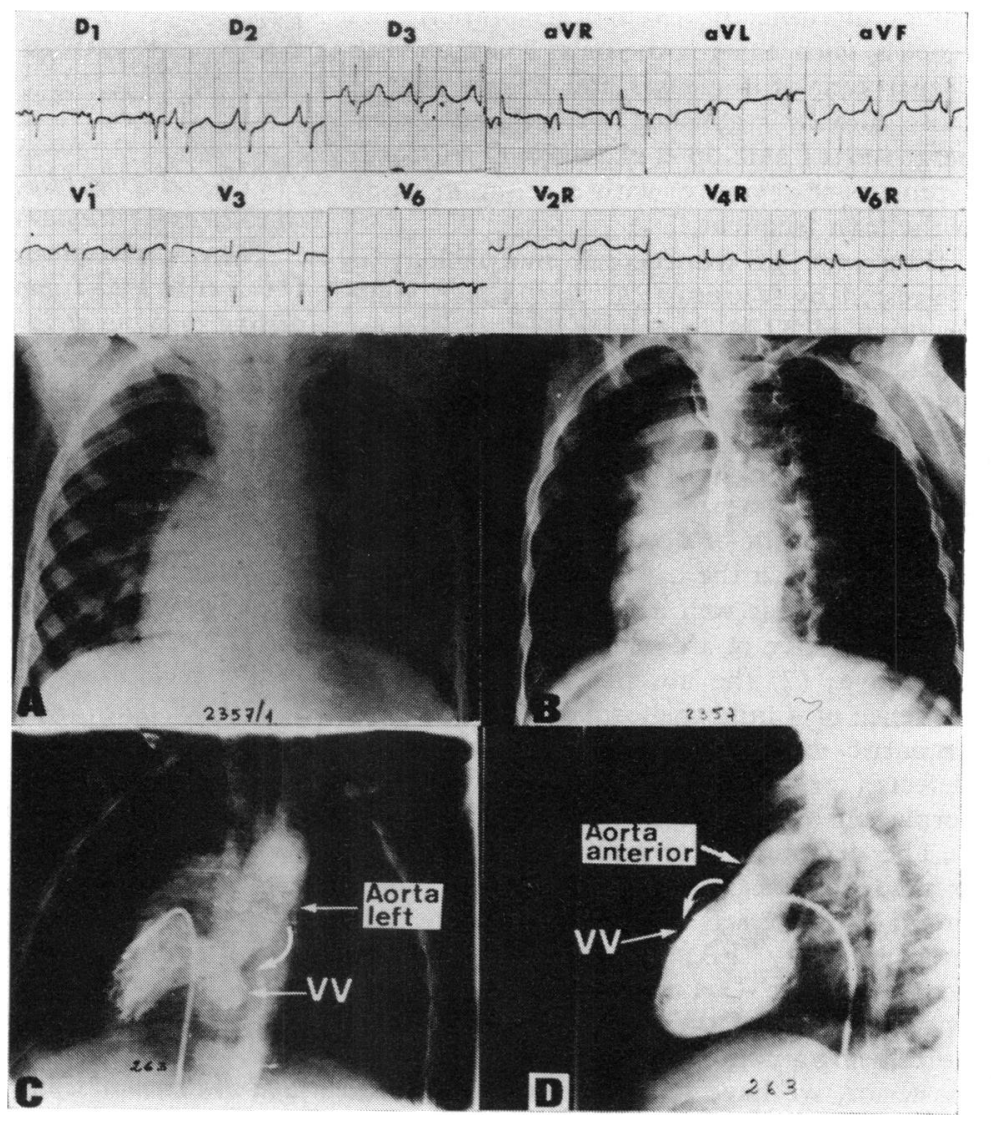


recorded over the right praecordium and morphologies pertaining to the 'right ventricle' recorded on the left, make us think of ventricular inversion (Fig. 5). In dextroversion, it is not always easy to locate the position of the ventricles by means of the electrocardiogram on account of complex malformations, which usually complicate this malposition (atresia of one of the atrioventricular orifices, single ventricle, etc), distorting the electrocardiogram to a considerable extent and making it difficult to recognize the morphologies peculiar to each of the ventricles.

Only a biplane angiocardiogram can determine with certainty the position of the ventricles; as soon as the truncoconal morphology is diagnosed, it is easy to deduce the position of the ventricles on the basis of embryological and anatomical knowledge (De la Cruz et al., 1971) which tells us that the ventral ventricle is situated immediately below the vessel which emerges anteriorly (when there are two vessels) or from the single trunk (in a case of common trunk).

The lateral position in the angiocardiogram shows which of the vessels is anterior, and the frontal position shows from which side the vessel in question emerges (Fig. 4 and 5). When the position of the anterior vessel is determined, the ventral ventricle will be immediately below the origin of this vessel;

- by correlating the position of the ventral ventricle in the frontal plane with the situs present in dextroversion (situs solitus), we will be able to determine whether there is concordance (without ventricular inversion) or not (with ventricular inversion), between the visceroatrial situs and the position of the ventricles.

Thus, when there is no TGA, the pulmonary artery is anterior, and this vessel emerges to the right of the mid-line and proceeds from right to left, backwards from the front and upwards from below, we know that the ventral ventricle is situated on the right and there is, therefore, no ventricular inversion. When the aorta is anterior it means that

- it is a case of TGA (Fig. 4); if this vessel emerges to the right it indicates that the ventral ventricle is situated on the right and there is no ventricular inversion; by the same reasoning in a case of a common trunk if the single vessel emerges from the right it means that the ventral ventricle is on the right and there is no ventricular inversion. When there is no TGA, the pulmonary artery is anterior and if this vessel is on the left of the mid-line and directed from left to right, from below upwards, and from the front backwards, we know that the ventral ventricle is on the left - an abnormal position in dextroversion - there is, thus, no concordance between the ventricular position and the situs of which the dextroversion is part (situs solitus); hence there is ventricular inversion. When there is TGA the aorta is anterior and if this vessel emerges on the left it indicates that the ventral ventricle is on the left and there is, therefore, ventricular inversion (Fig. 5). Similarly, in a case of common trunk if the vessel emerges on the left, it shows that the ventral ventricle is on the left and there is ventricular inversion.

Thus, once the truncoconal morphology has been established, we are able to determine the exact position of the ventricles by means of the angiocardiogram.

Unequal partition of truncoconus and lateral positions In dextroversion, truncoconal morphologies can occur (which is usual) complicated by unequal partition and/ or by lateral positions. When there are two vessels, the most common is an unequal partition at the expense of the pulmonary artery; this can be expressed anatomically as a mixed stenosis of the pulmonary artery (Fig. 4 and 5), and occasionally atresia. Fifty per cent of our complicated cases and 48 per cent of the published cases had pulmonary stenosis. This is one of the most frequent causes of cyanosis, physical incapacity, and, in general, intolerance to these malformations. A diagnostic approach is easy to make with the help of $x$-rays and can be confirmed by an angiocardiogram, which shows a narrow pulmonary artery anterior in cases without TGA; and posterior to the aorta in cases with TGA. The most frequent lateral position is dextroposition; in such cases the two vessels

FIG. 6 Scheme of the 6 possible combinations in dextrocardia with or without ventricular inversion, according to the three truncoconal morphologies.

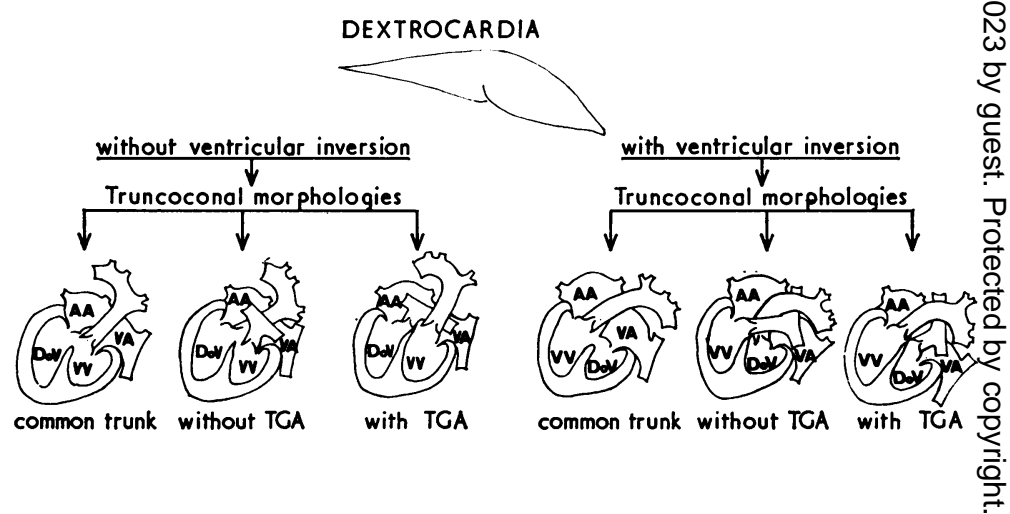


(Fig. 4), whether transposed or not, or a single trunk, emerge totally or partially from the ventral ventricle. The unequal partitions and/or lateral positions do not change the established rule, based on truncoconal morphology, for localizing the ventricles; it is sufficient to determine, by means of a biplane angiocardiogram, the side on which the anterior vessel or the common trunk is, in order to place on that side the ventral ventricle and correlate the position of this ventricle with the atrial position peculiar to the situs (situs solitus), thus enabling one to find out whether or not there is ventricular inversion.

Associated malformations As stated above, dextroversion is frequently associated with other disorders which, owing to their number and characteristics, cause a very complex malformation. To truncoconal morphologies and the position of the ventricles, as well as to unequal partition and/or lateroposition, are added malformations that influence the clinical picture. Septal defects are frequent; defects of the interatrial septum were found in 3 of our 10 cases with complications $(30 \%)$, and in 63 per cent of published cases, and defects of the interventricular septum in 80 and 84 per cent, respectively. Single ventricle is particularly frequent in dextroversions; a combination of single ventricle, TGA, and stenosis or atresia of the pulmonary artery is found in 64 per cent of these malpositions.

Other malformations associated with dextroversion are: atresia of one of the atrioventricular orifices, anomalous pulmonary venous drainage (partial or total), anomalies of drainage of the systemic veins, and persistent ductus arteriosus. The diagnosis of associated defects is sometimes difficult to make; septal defects are disclosed by catheterization; the angiocardiogram is essential for determining the presence of a single ventricle and other defects; some associated malformations can be suspected by the clinical data, $x$-rays, and electrocardiograms.

\section{Dextrocardia}

This is the most frequent of all cardiac malpositions: I8 out of our $4 \mathrm{I}$ cases $(44 \%)$. Being the most common, it is the least complicated of all cardiac malpositions; it is the best known and the one giving rise to least confusion; its nomenclature is the simplest and has few variants; it is known under the name of mirror-image dextrocardia in situs inversus. We can define dextrocardia as the
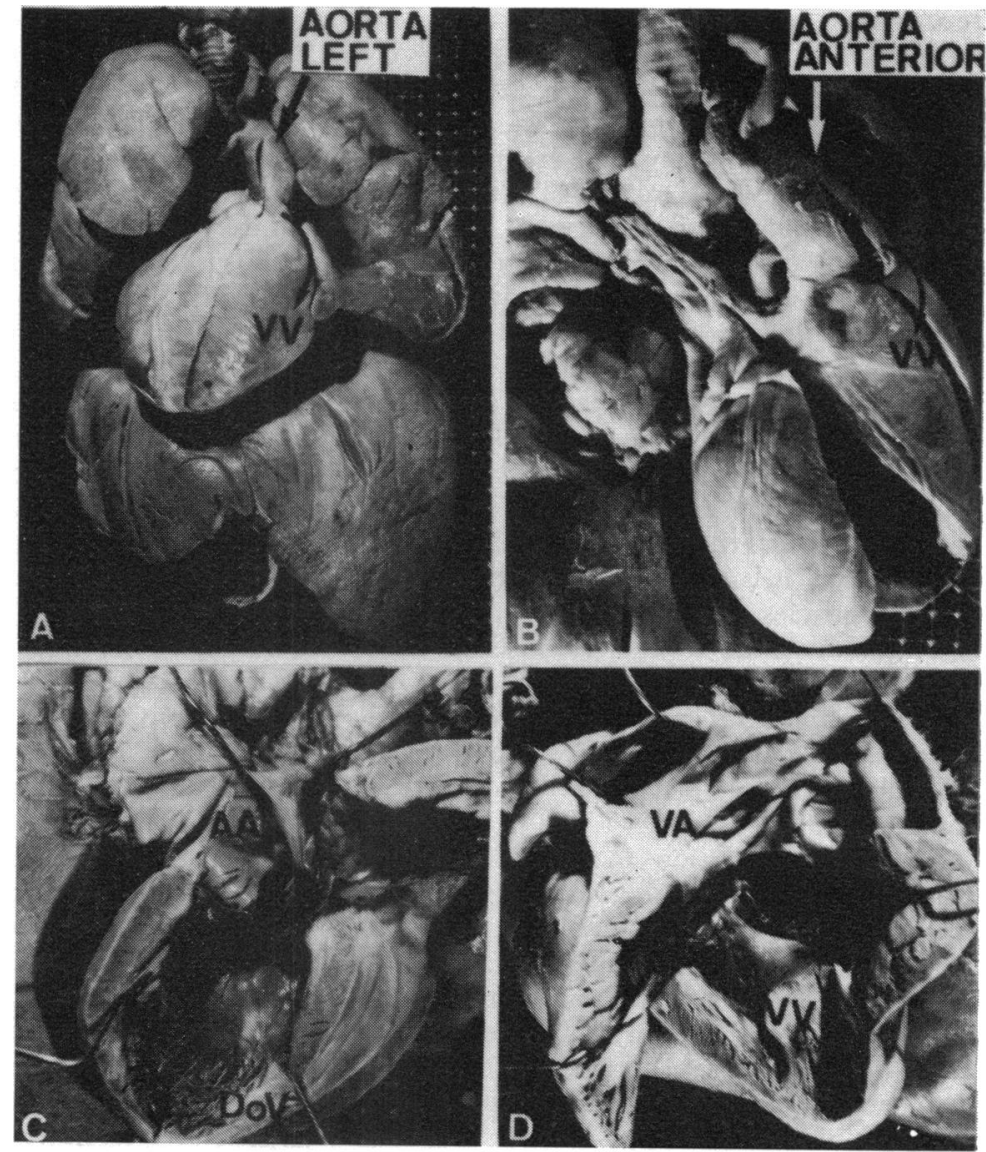

FIG. 7 Necropsy specimen of one of our cases of situs inversus. In $A$, the greater lobe of the liver can be seen to the left, the heart is in the right, and the apex is directed toward the right: dextrocardia. In $B$, a lateral view, the aorta is anterior and the pulmonary artery is narrow and posterior. The aorta emerges to the left $(A)$ from the ventral ventricle which is situated to the left; this situation is normal, concordant for this ventricle in situs inversus; therefore there is not ventricular inversion.

In $C$, there is evidence of the normal relation of the arterial atrium with the dorsal ventricle and, in $D$, the relation of the venous atrium with the ventral ventricle. This is a case of situs inversus with dextrocardia, without ventricular inversion, with TGA and pulmonary stenosis (unequal partition at the expense of the pulmonary artery). A complete atrioventricular canal was an associated defect, as it can be seen in $C$ and $D$. 
position characterized by situs inversus (greater lobe of the liver, the ventral atrium, and the

- suprahepatic segment of the inferior vena cava on the left), the heart in the right hemithorax, and the apex directed towards the right.

Dextrocardia is seldom associated with other malformations; ro out of our 18 cases $(55 \%)$ showed no complications. Hanson and

- Tabakin (196I) found that 90 per cent of dextrocardias were not accompanied by other

- defects. This high incidence of normal hearts in dextrocardia probably proves that dextrocardia is a 'normal position' of the heart in a situs inversus of which it is a part; Grant (I958) gives 5 per 1000 for complicated - dextrocardias, which constitutes a similar incidence for congenital malformations of the - heart for the population in general. Those complicating dextrocardias are usually sim-

ple and acyanotic; 56 per cent of our cases showed mild, acyanotic malformations; complex, severe, cyanotic ones are much less common.

Truncoconal morphologies occur in dextrocardia in the three basic types seen above: (a) without $T G A$, i.e. with vessels in a normal relation; (b) with $T G A$, i.e. the aorta in an anterior position emerging from the ventral ventricle and the pulmonary artery posterior to the aorta, both vessels being parallel;

- (c) with a common trunk, where a single vessel emerges from the heart. Any of the three types of morphology can be found in dextrocardia, but type (a) without TGA is the most common ( $78 \%$ of our cases); type (b) cases with TGA are less frequent $(22 \%)$; the least common is type (c) with a common trunk. These statistical data differ from those given for dextroversion, where the most common type is the one with TGA.

Certain facts obtained through routine cardiological examination enable us to suspect the type of truncoconal morphology: (i) statistical data of the frequency of the normal relation of vessels in dextrocardia; (ii) the absence of cyanosis allows us in most cases

- to suspect type (a) without TGA; the opposite reasoning would not be valid, as cyanosis could indicate cyanotic defects without TGA; (iii) clinical and radiological data suggesting TGA can help by indicating this type of defect; in TGA with pulmonary stenosis, clinical differentiation from tetralogy of

- Fallot is difficult, as it is in normally situated hearts; (iv) clinical and radiological data suggestive of a common trunk (Espino Vela, 1959) are applicable to diagnostic suspicion of this type of truncoconal morphology in dextrocardia.

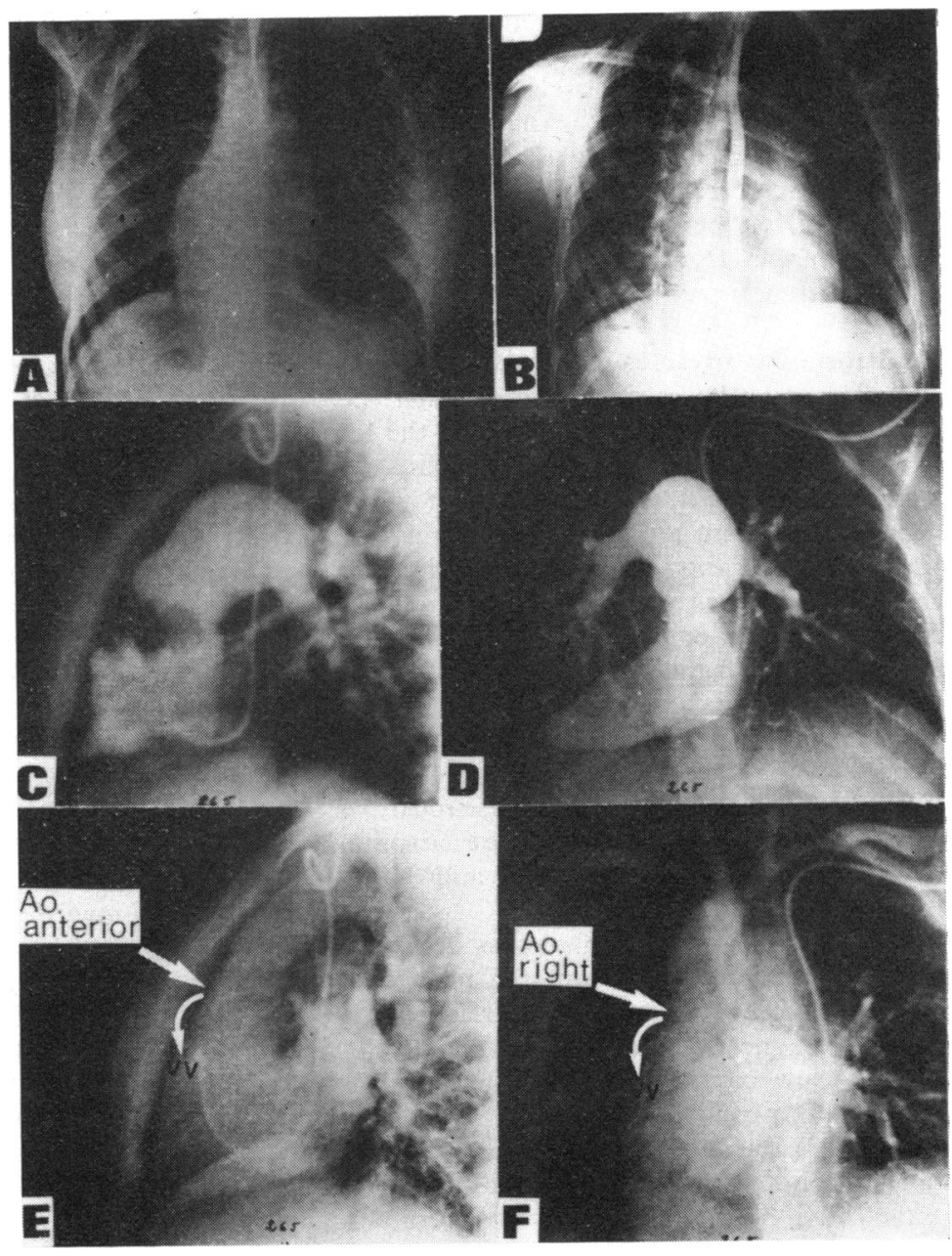

FIG. 8 Radiological and angiocardiographic studies in a case of situs inversus. In $A$ and $B$ it is only possible to determine from the shadow of the liver, the position of the heart, and the direction of the apex, that this is a case of dextrocardia. In C, the pulmonary artery is seen dilated, and in $D$ the vessel emerges in a central position from a ventricle with a peculiar shape which is characteristic of the dorsal ventricle in cases of ventricular inversion. In $E$, the aorta is anterior in the lateral projection and in $F$, the frontal projection, the aorta is to the right, which indicates that the ventral ventricle is to the right, a position which is abnormal, not concordant for that ventricle in situs inversus, and therefore, there is ventricular inversion. This is a case of situs inversus with dextrocardia, ventricular inversion, TGA, and there was an atrial septal defect as an associated malformation, which was successfully treated by operation. 
The positive diagnosis of truncoconal morphology is made by means of a biplane angiocardiogram: if the vessels cross in space and the pulmonary artery is the anterior vessel, we are dealing with type (a) without TGA; if the vessels are parallel, the aorta being anterior, it is type (b) with TGA; if a single vessel emerges from the heart, it is type (c) with a common trunk.

Position of ventricles In dextrocardia the ventricles may be in a normal position, i.e. with the ventral ventricle situated on the left and the dorsal ventricle on the right, this being in agreement with the situs of which dextrocardia forms a part (situs inversus). An alternative is ventricular inversion, i.e. the ventral ventricle situated on the right and the dorsal ventricle on the left, a position in discordance with the situs. Two of our complex dextrocardias $(22 \%)$ had ventricular inversion, which is much less frequent in dextrocardias than in dextroversions; in the published material too, cases of dextrocardias with ventricular inversion are rare (EspinoVela et al., 1959; Payne, Ellis, and Hunt, I96I; Van Praagh et al., 1964).

The 3 truncoconal morphologies described above, which can be encountered in dextrocardia with or without ventricular inversion, raise the diagnostic possibilities to 6 (Fig. 6).

There are certain data that help in the diagnosis of suspected ventricular positions: they are (i) the low incidence of ventricular inversion in dextrocardia, which is important statistically. (ii) The presence of morphologies corresponding to variations of potential of the ventral ventricle in the left praecordium (V2 to V6), and morphologies corresponding to the dorsal ventricle in the right praecordial leads ( $44 R$ to V6R) indicate that there is no ventricular inversion (Fig. I); on the other hand, morphologies corresponding to variations of potential peculiar to the dorsal ventricle in the left praecordium and morphologies corresponding to the ventral ventricle in the right praecordium indicate the possibility of ventricular inversion. (iii) The presence of ventricular inversion, suggested by an electrocardiogram, presupposes TGA; published cases of ventricular inversion without TGA in situs inversus are exceptional, only 2 having been published so far (EspinoVela et al., 1970).

A positive diagnosis of the position of the ventricles can only be made by means of a biplane angiocardiogram. As soon as the truncoconal morphology has been diagnosed, we can deduce the position of the ventricles by following the rule established previously: the ventral ventricle is situated immediately below the vessel emerging anteriorly (when there are two vessels) or from the single trunk (when there is a common trunk). The angiocardiogram in the lateral position will show which is the anterior vessel and the frontal projection shows on which side this vessel is placed; this method enables us to locate the ventral ventricle and shows whether or not there is ventricular inversion by correlating the position of this ventricle to that corresponding to the situs inversus. Thus, when we are dealing with type (a) without TGA, the pulmonary artery is anterior; if this vessel emerges from the left of the mid-line it shows that the ventral ventricle is on the left, which is the correct position for a situs inversus; there is, therefore, no ventricular inversion; if we are dealing with type (b) with TGA, the aorta is anterior and by emerging on the left it indicates that the ventral ventricle is on the left and there is no ventricular inversion (Fig. 7). Similarly, when dealing with type (c) with a common trunk, if the single trunk emerges from the left, it shows that the ventral ventricle is on the left. When there is ventricular inversion, the anterior vessel (whether pulmonary artery or aorta) or the common trunk emerge from the right of the mid-line, showing that the ventral ventricle is on the right (Fig. 8), and there is, therefore, a lack of correlation between the position of this ventricle and the situs of dextrocardia (situs inversus).

\section{Unequal partition of truncoconus and} lateral positions The various truncoconal morphologies may be complicated by an unequal partition of the truncus conus (when

FIG. 9 Scheme of the 6 possible combinations in laevoversion, with and without ventricular inversion, according to the three truncoconal morphologies.

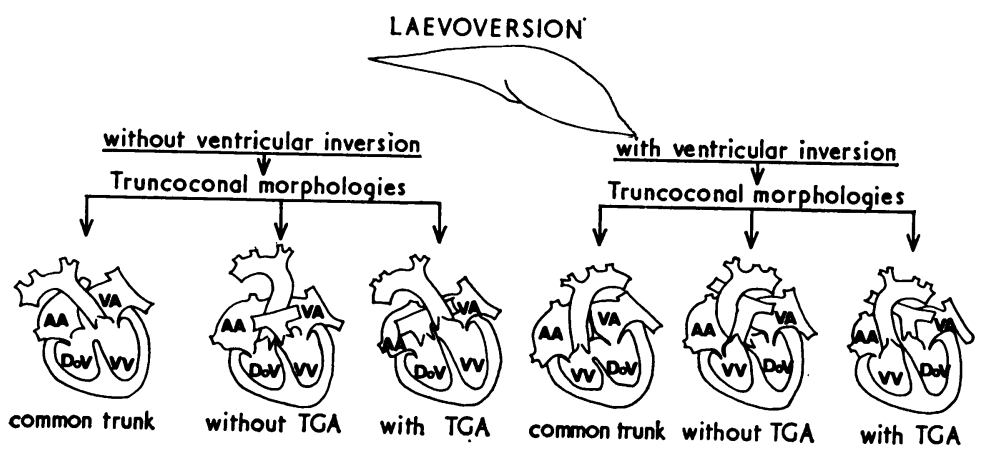


there are two vessels) and/or by lateral positions. The most frequent of the unequal - partitions is at the expense of the pulmonary artery, i.e. stenosis of the pulmonary artery. 'Dextroposition' is the most frequent among lateral positions, i.e. both vessels (or the single trunk) emerging totally or partially from the ventral ventricle, wherever this ventricle happens to be situated.

Of our 9 complicated dextrocardias, 3 showed mixed pulmonary stenosis $(33 \%)$.

- This complication is one of the causes of cyanosis and unavoidably implies a ventri- cular septal defect (De la Cruz and Da Rocha, 1956). The diagnosis of an unequal partition at the expense of the pulmonary artery is easily made by $x$-rays which show a decreased pulmonary circulation. The diag-

- nosis is confirmed by an angiocardiogram which shows a narrow and anterior pulmonary

- artery in type (a) without TGA; narrow and posterior in type (b) with TGA; it also indicates the type and degree of lateral position. Unequal partition and/or lateral position do - not alter the rules established for locating the ventricles.

- Associated malformations We have already mentioned the low incidence of associated malformations in dextrocardia, which, when present, are usually mild. The diagnosis

- does not vary much, as signs and symptoms are similar to those of identical malformations found in a normally situated heart; the only difference is in the location of the cardiac sounds and of murmurs, when they exist. Keeping in mind the particular position of the cardiac chambers and great vessels will help in arriving at a suitable interpretation. An electrocardiogram is helpful, as it shows the type of overloading, enlargement of cavities, bundle-branch block, etc. Moreover, $x$-rays give us an idea of the pulmonary circulation. Catheterization and/or angiocardiography show associated malformations. Of our 9 complicated cases, 4 showed malformations of the interatrial septum ( 2 had a

a single atrium, I had ostium primum, and I had ostium secundum); among cyanotic defects, we had 2 cases of atresia of the venous atrioventricular orifice.

\section{Laevoversion}

- This is the least frequent of the malpositions ( $7,17 \%$ of our $4 \mathrm{I}$ cases). It has been called by other names, such as laevocardia or single laevocardia. This malformation has led to most erroneous diagnoses, owing to the fact that it is usually associated with other very

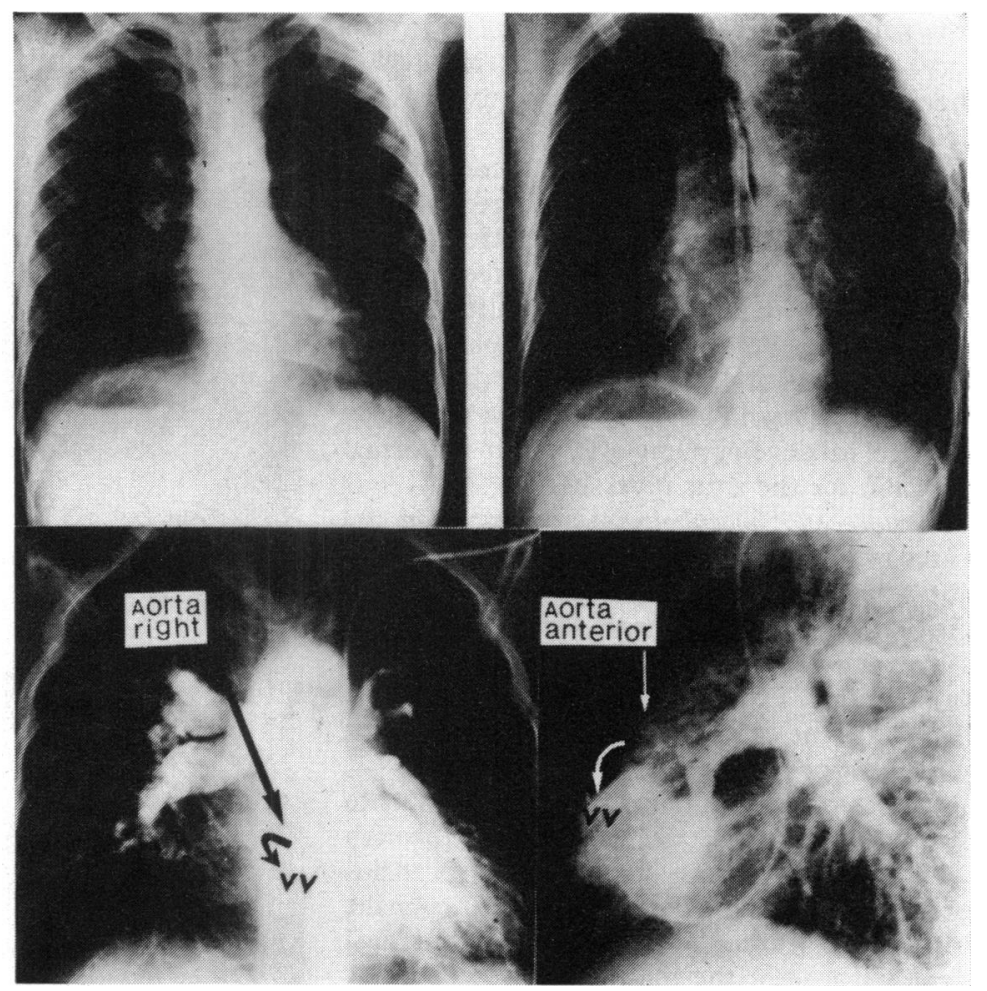

FIG. Io Radiological and angiocardiographic studies in a case of situs inversus. The plain films show the shadow of the liver to the left, the heart in the left hemithorax, and the apex directed toward the left: laevoversion, the decrease in pulmonary flow suggests pulmonary stenosis. The angiocardiogram in the lateral projection shows that the aorta is anterior and the pulmonary artery is posterior and narrow. In the frontal projection the aorta is to the right, which indicates that the ventral ventricle is to the right, this ventricular position is abnormal, not concordant with situs inversus, therefore there is ventricular inversion.

This is a case of situs inversus with laevoversion, ventricular inversion with TGA, pulmonary stenosis, and ventricular septal defect.

complex malformations, which have produced an unsuitable nomenclature and mistaken interpretations, not conforming to modern anatomoembryological knowledge; as a result, the true incidence of this malposition is not known and many cases of so-called laevoversion are normally-situated hearts with ventricular inversion, while others are heterotaxias. Our definition of laevoversion is a malposition characterized by situs inversus (greater lobe of the liver, venous atrium, and 
the suprahepatic segment of the inferior vena cava on the left), with the heart in the left hemithorax and the apex directed towards the left.

In all our cases laevoversion is complicated by very severe and complex congenital malformations, all of them cyanotic from birth; only a few cases have been reported without cyanosis or with mild associated malformations (Rosenbaum, Pellegrina, and Treciokas, 1962). This is probably due to the fact that laevoversion in situs inversus indicates a very early change in development and is an abnormal position for the situs inversus.

Truncoconal morphologies are found in the three types described above; (a) without TGA, (b) with $T G A$, and (c) with a common trunk. Any of the three morphologies is met in laevoversion; 3 of our cases were of type (a) without TGA, and 4 of Type (b) with TGA. Of the cases reported, over 50 per cent were of type (b) with TGA.

There are certain data that might lead us to suspect the truncoconal morphology through clinical examination, $x$-ray, and electrocardiogram, but such a diagnosis is difficult to make in this malposition, except for the statistical data, because of the high frequency of TGA in laevoversion. Only a biplane angiocardiogram will show with certainty the truncoconal morphology, as we have already stated; if the two vessels cross and the pulmonary artery is anterior, we have type (a) without TGA; if the two vessels are parallel, the aorta being anterior, we have type (b) with TGA, and if a single vessel emerges from the heart, we are dealing with type (c), a common trunk.

Position of ventricles The ventricles may be normally situated in laevoversion, i.e. the ventral ventricle on the left and the dorsal ventricle on the right, a position in concordance with the situs of which laevoversion forms a part (situs inversus). The other possibility is ventricular inversion, i.e. the ventral ventricle on the right and the dorsal ventricle on the left, a ventricular position in discordance with the situs of which laevoversion is part. Two of our cases showed ventricular inversion. It is difficult to find out the percentage of ventricular inversion in published cases of laevoversion because of the confusion in some of the descriptions.

The three truncoconal morphologies may be found in laevoversion with or without ventricular inversion. There are 6 diagnostic possibilities (Fig. 9).

The position of the ventricles in laevoversion is difficult to suspect from clinical

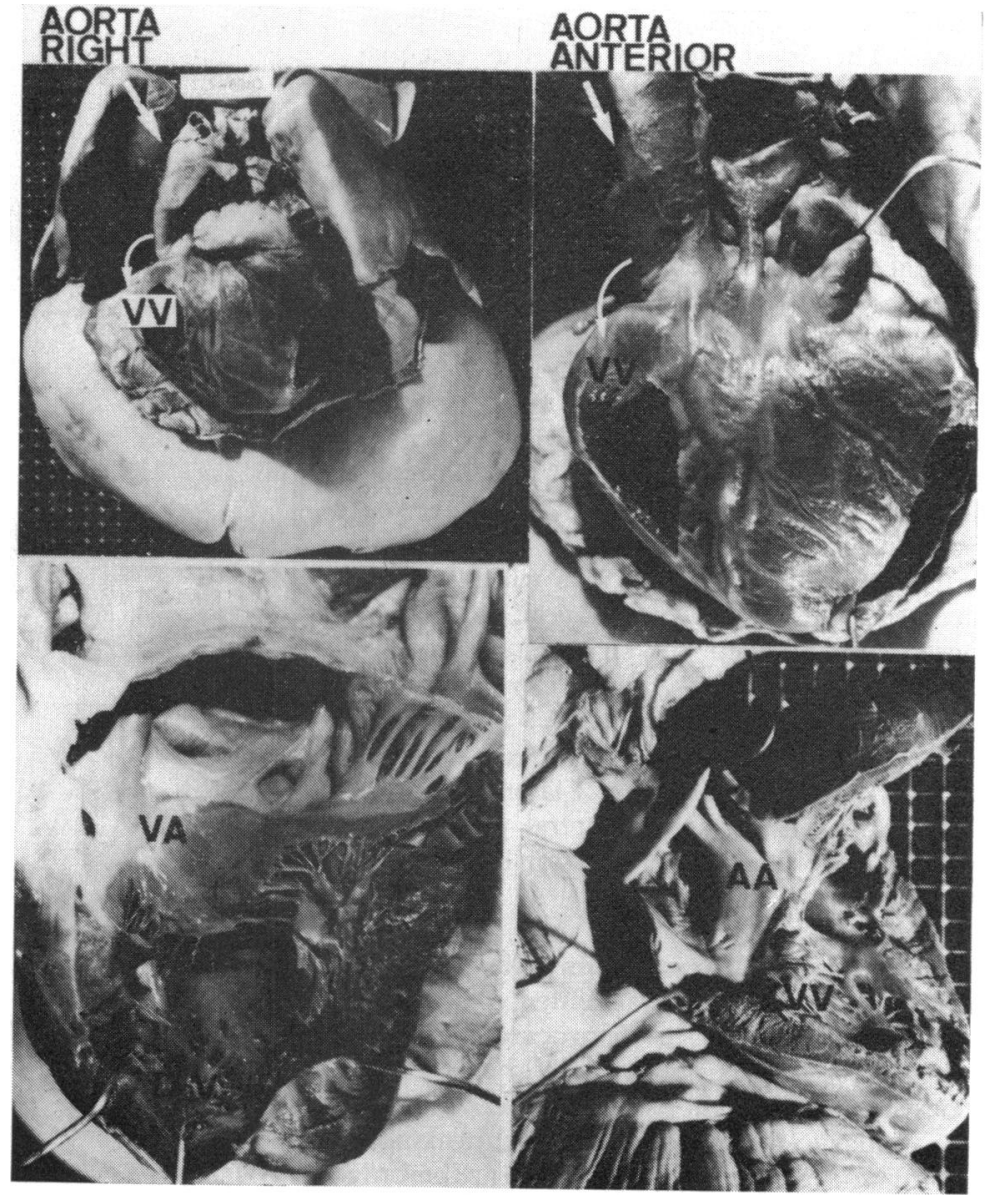

FI G. I I Necropsy specimen of a case simi-

lar to that of Fig. 10. In the left upper picture, the greater lobe of the liver and the heart can be seen on the left and the apex is also directed towards the left: situs inversus with laevoversion. The right upper is a left lateral view of the heart, the aorta is anterior, and the pulmonary artery is posterior and narrow. In both lower pictures the heart has been opened, in order to show the relation of the venous atrium with the dorsal ventricle and of the arterial atrium with the ventral ventricle, which means ventricular inversion. Besides, there was a ventricular septal defect. This is a case of situs inversus with laevoversion, ventricular inversion with $T G A$, pulmonary stenosis, and ventricular septal defect.

data, $x$-rays, or electrocardiograms for the following reasons: (i) we do not know with certainty the incidence of ventricular inversion in laevoversion, (ii) the examination of unipolar morphologies of electrocardiograms is of relative value for locating the ventricles 
on account of the high incidence of a single ventricle in laevoversion (Fig. 2). It is known (Anselmi et al., 1968) that in this type of defect the electrocardiographic morphologies vary considerably and bear no relation to the anatomical type of single ventricle. (iii) The high incidence of complex defects associated with laevoversion makes it difficult to suspect the position of the ventricles.

The only positive diagnosis of ventricular position is arrived at by means of the biplane angiocardiogram, which determines, in the lateral projection, which of the vessels is anterior and, in the frontal projection, from which side this vessel emerges, thus indicating logically the position of the ventral ventricle; by correlating the position of this ventricle with that corresponding to the situs inversus, we find out whether or not there is ventricular inversion. Thus, if the anterior vessel (pulmonary artery in type (a) without TGA, the aorta in type (b) with TGA, or the common trunk in type (c)) emerges from left, it will indicate that the ventral ventricle is situated on the left, the normal position for the situs; if, on the contrary, the anterior vessel or the single trunk emerges from the right, it means that the ventral ventricle is on the right and, therefore, there is discordance between the ventricular position and the situs; so there is ventricular inversion (Fig. IO and II).

Unequal partition of truncoconus and lateral positions These are usual in laevoversion and complicate truncoconal morphologies. Mixed pulmonary stenosis (Fig. Io and II) is particularly frequent (unequal partition at the expense of the pulmonary artery); 6 of our 7 cases $(86 \%)$ showed stenosis or atresia of the pulmonary artery, which is one of the causes of cyanosis and intolerance to such malformations. It is easy to suspect the diagnosis by plain films (Fig IO) and to confirm it by an angiocardiogram; the latter will show whether there is lateral position complicating any of the truncoconal morphologies.

An unequal partition and/or lateral positions do not alter the rule established for diagnosing the position of the ventral ventricle by means of the biplane angiocardiogram.

Associated defects Laevoversion is associated with very complex defects which add to the severity of the prognosis. Interatrial and interventricular septal defects are frequent; of our 7 cases, 3 had interatrial and 4 interventricular septal defects ( 3 of the latter had a single ventricle). Campbell and Deuchar
(1965), found that 75 per cent of their cases had ventricular septal defects, of which 25 per cent were single ventricles. Other common defects are atresia of the atrioventricular orifices and anomalies of pulmonary and systemic venous return. In some cases it is easy to suspect the associated malformations; a single ventricle (Fig. 2), by the presence of any of the 3 types of electrocardiographic patterns common in this cardiopathy (Anselmi et al., I968); obvious deviation of the $\hat{A} Q R S$ to the left or right can suggest atresia of one of the atrioventricular orifices; $x$-rays can show signs of anomalous pulmonary venous drainage. Catheterization and angiocardiogram will confirm with certainty associated defects.

\section{References}

Anselmi, G., Muñoz, S., De la Cruz, M. V., Pisani, F. de, and Blanco, P. (1968). Diagnosis and classification of single ventricle. Report on seventeen cases with an anatomoembryologic discussion. American fournal of Cardiology, 21, 813 .

Anselmi, G., Muñoz, S., Machado, I., Blanco, P., De la Cruz, M. V., and Bello, M. (1970). Systematization of malpositions of the heart and complex associated malformations. Clinical diagnosis of dextrocardias and laevoversions (abstract.) VI World Congress of Cardiology. Cardiovascular Research, Suppl., 64.

Ayres, S. M., and Steinberg, I. (1963). Dextrorotation of the heart. Circulation, 27, 268.

Berri, G. G. (1958). Dextrocardias y Levocardias. Talleres Gráficos, S.R.L., Didot, Buenos Aires.

Calderón, J., Marquez, J., Cerezo, L., Sanchez, E., Fernandez-Nespral, V. A., and Torrecilla, I. (1965). Dextrocardias con auriculas 'in situ solito'. Revista Española de Cardiologia, 18, 40.

Campbell, M., and Deuchar, D. C. (1965). Dextrocardia and isolated laevocardia. I. Isolated laevocardia. British Heart fournal, $27,69$.

De la Cruz, M. V., Anselmi, G., Muñoz-Castellanos, L., Nadal-Ginard, B., and Muñoz-Armas, S. (197I). Systematization and embryological and anatomical study of mirror-image dextrocardias, dextroversions, and laevoversions. British Heart fournal, 33, 841 .

De la Cruz, M. V., and Da Rocha, J. P. (1956). An ontogenetic theory for the explanation of congenital malformations involving the truncus and conus. American Heart fournal, 51, 782.

De la Cruz, M. V., Espino-Vela, J., Attie, F., and Muñoz, L. (1967). An embryologic theory for ventricular inversions and their classification. American Heart Fournal, 73, 777.

Espino Vela, J. (1959). Malformaciones Cardiovasculares Congénitas. Ediciones del Instituto Nacional de Cardiología, México.

Espino-Vela, J., De la Cruz, M. V., Muñoz-Castellanos, L., and Attie, F. (1970). Ventricular inversion without transposition of the great vessels in situs inversus. British Heart fournal, 32, 292.

Espino-Vela, J., Portillo, B., Anselmi, G., De la Cruz, M. V., and Reinhold, M. (1959). On a variety of the 'corrected' type of transposition of the great vessels associated with dextrocardia: a study of two cases with autopsy report. American Heart fournal, 58, 250. 
Grant, R. P. (1958). The syndrome of dextroversion of the heart. Circulation, 18, 25.

Hanson, J. S., and Tabakin, B. S. (196I). Primary and secondary dextrocardia. Their differentiation and the role of cineangiocardiography in diagnosing associated congenital cardiac defects. American fournal of Cardiology, 8, 275.

Payne, W. S., Ellis, F. H., Jr., and Hunt, J. C. (196I). Congenital corrected transposition of the great vessels with situs inversus and dextrocardia. Report of surgical repair of associated defects in a patient with pulmonary stenosis, interatrial communication and persistent 'left' superior vena cava. American fournal of Cardiology, 8, 288.

Portillo, B., Anselmi, G., Sodi-Pallares, D., and Medrano, G. A. (1959). Importance of the unipolar leads in the diagnosis of dextrocardias, levocardias, dextropositions, and dextrorotations. American Heart fournal, 57, 396.

Rosenbaum, H. D., Pellegrino, E. D., and Treciokas, L. J. (1962). Acyanotic levocardia. Circulation, 26, 60.
Shaher, R. M., and Johnson, A. M. (1963). Isolated laevocardia and isolated dextrocardia: pathology and pathogenesis. Guy's Hospital Reports, 112, 127.

Sodi-Pellares, D., Bisteni, A., Fishleder, B. L., and Medrano, G. A. (1959). Importance of the unipolar morphologies in the interpretation of the electrocardiogram: the theoretical basis of the unipolar morphologies and its correlation with vectorial analysis with cardiac activation, and with the potential variations at the epicardial surface of the heart. American Heart fournal, 57, 590.

Van Praagh, R., Van Praagh, S., Vlad, P., and Keith, J. D. (I964). Anatomic types of congenital dextrocardia. Diagnostic and embryologic implications. American fournal of Cardiology, 13, 510.

Requests for reprints to Dr. Guillermo Anselmi, P.O. Box 59019, Caracas 104, Venezuela, South America. 